\title{
Expanding the Boundaries of FIB-SEM Technology: Developments for Best Application Results
}

\author{
I. Schulmeyer, M. Kienle \\ Carl Zeiss Microscopy GmbH, Carl-Zeiss-Straße 22, 73447 Oberkochen, Germany
}

Increasing demands in 3D-imaging, -analytics and Nanofabrication require FIB-SEM Instruments with superior properties, quality and flexibility. The latest developments in Carl Zeiss FIB-SEM technology address this demands and form an ins trument that is well prepared for numerous today and future applications.

FIB-tomography allows the volumetric reconstruction at a sub-10nm resolution. The trend in Material and Life Sciences is to aquire increasingly large volumes at higher voxel resolution. This leads to a better understanding of compound materials, brain tissues, semiconductors and other samples. Achieving large images at high resolution requires a special design of the electron optics. A non-uniform magnetic field on the sample surface limits the field of view due to distortions in the outer regions. Latest developments of the Carl ZEISS SEM column leads to a minimum magnetic field and thereby to minimum distortions, allowing large field of view imaging without stage movement and stitching. A limitation in large volume FIB-tomography is the time needed to acquire this huge datasets. To reduce the acquisition time it is necessary to perform milling of the slices and imaging at the same time. The acquisition of up to 4 detector signals at the same time leads to maximum information in the least time. Intelligent software algorithms and complete workflow support of ATLAS3D allow to coll ect only the relevant information and to improve z-resolution.

Throughput for various applications can also be achieved by faster FIB-milling. This requ ires higher FIB currents. Though, FIB resolution and low kV Performance shall be maintained. The latest column design offers currents up to $100 \mathrm{nA}$ with superior spot profiles while achieving best-in-class resolution at lower currents and excellent low $\mathrm{kV}$ performance.

Modern FIB-SEM sy stems cover not only the typical application range but are also able to host and integrate numerous instruments and components for advanced experiments. The Zeiss FIB-SEM allows integration of many $3^{\text {rd }}$ party systems like heating, cooling and tensile stages, SIMS, EBIC and CL, several manipulators, e.g. for probing and liftout, a Laser option for massive ablation and many more. A modern API interface that is open to the user allows the seamlessly integration into the FIB-SEM.

The new SEM lens design offers the optimum conditions for high resolution imaging and high current analytics in one tool. The performance at high current conditions were improved leading to a significant reduction of time for analytics. Changing imaging modes and optical parameters like probe current, acceleration voltage or working distance can be done in a fast and easy way never seen before on a FIBSEM. 


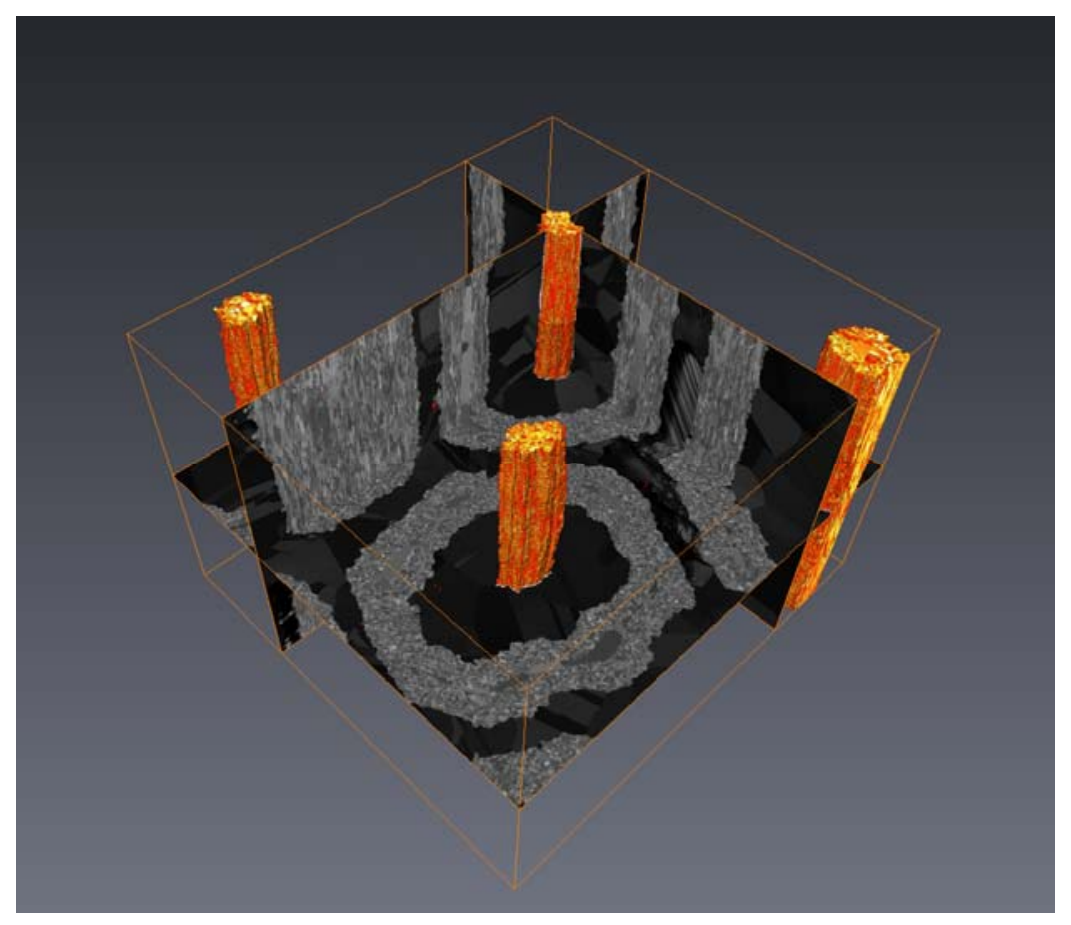

Figure 1. Nb3Sn multi-filament superconducting cable acquired with ATLAS3D. The volume of $25 \times 23 \times 17 \mu \mathrm{m}$ comprises 1700 slices. The images were acquired using simultaneous milling and imaging with the EsB detector. The 3D reconstruction shows the complex fine grained microstructure. Materials contrast as well as grain orientation contrast can be observed in the superconductor phase as well as in the $\mathrm{Cu}$ matrix. (Sample Courtesy of Dr. Marco Cantoni, EPFL, Switzerland) 